\title{
Distinctive parameters of action of stem cells and modulatory microenvironmental microcirculation in gliomagenesis
}

\author{
Lawrence M. Agius
}

Department of Pathology, Mater Dei Hospital, University of Malta Medical School, Msida, Malta; kcchou@gordonlifescience.org

Received 12 October 2012; revised 13 November 2012; accepted 15 December 2012

\begin{abstract}
Definition of the malignant transformation event is central to a distinction between neural stem cells and cancer stem cells. In such manner, the descriptive analysis of various tumors such as gliomas would allow for the distinction of genetic injury and probably epigenetic events that transform gene transcription pathways. Hypoxia is a major conditioning influence acting on stem cell niche microenvironments that evolve in terms particularly of micro-vascular dynamics. The incremental involvement of entire fields of cancerization allows for the establishment of permissive conditions of repetitive nature and within the contextual involvement of multiple clones of injured cells that condition, in turn, the stem cell niche. In view of the establishment of progressive malignant change, it is significant to view the cancerization as an integral involvement of both sequential and concurrent events in defining the roles of stem cells and cancer stem cells in terms of a primal process of dedifferentiation beyond simple markers of morphologic transformation.
\end{abstract}

Keywords: Stem Cells; Gliomagenesis; Microcirculation; Malignant Transformation

\section{INTRODUCTION}

Derivative recombination of various facets in the characterization of gliomas indicates a restricted array of features that would tend to categorize malignant tumors in terms of biology of stem cells and of cancer stem cells. The acquistion of stem-like traits might promote glioma initiation, growth and recurrence [1] The field of stem cell research applies also to regenerative medicine [2]. Hierarchical organization of the proliferative and differential attributes of a glioma indicates a strict conforma- tional profile that includes the distribution and further multiple redistributions of profiles of activity of the lesion. It is with a series of sequential steps in accumulative potential that genetic or karyotypic abnormality assumes a prominent idealization of neoplasms beyond complex systems of dysregulation. The rates of existence of glioma cancer stem cells increase proportionally with rise in WHO grade of glioma [3]. Human bone marrowderived mesenchymal stem cells inhibit human glioma growth through downregulation of PDGF/PDGFR axis [4]. Particular specificity in the carcinogenesis and further to the upset of the oncogene/suppressor gene equilibrium there would constitute a series of active sites or domains as integrally composed transformational events.

\section{REVIEW}

\subsection{Stem Cells}

Demands of stem cell characterization include a realization of an undifferentiated stem cell profile in the subsequent utilization of injury and hypoxia, in particular, in regulatory biology of the stem cell niche. Including both manners of biologic attributes of stem and progenitor cells would comprise the evolutionary course of dedifferentiation.

Glioma-initiating cells are responsible for resistance to chemotherapy and radiation and for recurrence [5].

Such dedifferentiation in gliomas includes the derivation of onset pathways reflected in excessive cellular proliferation and the establishment of non-apoptosis. The orphan nuclear receptor TLX promotes brain tumorigenesis [6].

Specificity of malignant transformation comprises the constitutional insertion of stem cells that directly promote the distribution of injurious agents that mutagenically compromise the mechanisms of normal cell differentiation. Innate brain region neural stem cell heterogeneity is implicated in the patterning of gliomagenesis [7].

It is especially significant to consider stem cells as the 
source of biologic viability of gliomas in terms of the consequent and sequential developments of molecular pathways in cancerization. MicroRNAs play an important role in renewal and differentiation of cancer stem cells [8].

\subsection{Malignancy}

Cancer stem cells are modulated source for the institutional emergence of malignancy in addition to the system attributes of proliferating cells that diffusely infiltrate. A significant field cancerization phenomenon includes a spread of diffusible factors in operative modulation of transcriptional control of various genes. Epigenetic modulation is implicated in the crosstalk of cancer cells with their environment [9].

\subsection{Dedifferentiation}

Such pathways include epigenesis that arises in context of the variable parameters induced by the microenvironment and in particular by the niche microvasculature. The apoptotic ligand TRAIL holds promise as cancer gene therapy [10].

Intentional synthesis of undifferentiated stem cell nature includes the promotion of systems that include both autocrine and paracrine influence on stem cells.

The strict reappraisal of transforming integralization of injuries allow for the promotion of distribution dynamics underlying active glioma infiltration of adjacent fields of cancerization. It is further to such promotional events that malignant transformation contributes to the systemic implications of subsequent development of the malignant potential for dedifferentiation of the tumor cells.

The anti-oxidant enzyme status appears a significant feature of heterogeneity of glioblastomas [11].

In a strict context of progression, the institution of multiple profiles of superimposed change and of field involvement evolves as systems of biologic derivation of stem cell attributes. Deregulation of the retinoblastoma, phosphoinositide-3 kinase and p53 pathways characterizes gliomas [12].

Complex categorization of the carcinogenic influences arises as composite determination of injury that assumes the regulatory pathways of constitutional change in terms of proliferative activity. Cancer stem cells are derivative dysfunctionality that is expressed as dedifferentiation during the stage of carcinogenesis.

In view of paramount integration of various forms of injurious events, there is instituted a strong tendency in assuming stem-cell profiles that are self-promotional. CD133, despite its association with stem cells, remains to be defined as a causal relationship to tumorigenesis
[13].

\subsection{Dysregulation}

It is in terms of system pathways that accumulative phenomena of genetic damage and of epigenetic dysregulation assume multi-parallel consequences as reflected in such stem-cell biologic activities. Our picture of the many interactions between tumor and parenchymal cells is incomplete [14].

Considerable expansion of dimensions in glioma dedifferentiation accounts for tumor biology that is both derivative function and also promotional pathway for assumed stem-cell potentiality [15].

It is in terms of an essential field distribution of abnormal profiles of injury that the whole complex array of dysregulations accounts for the establishment of the dedifferentiation process central to the development and progression of carcinogenesis. The stemness phenotype model proposes that all glioma cells possess stem cell properties with also microenvironment-induced modulation [16].

\subsection{Drivers of Carcinogenesis}

The drivers of carcinogenesis are constituted by the integral dedifferentiation phenomenon that includes the assumption of multi-potential for subsequent assumption of excessive proliferative rate and for the integral development of microenvironmental characterization.

As such, incremental aspects of a stem-cell characterization include the overall field cancerization in gliomagenesis. Alterations in microRNA expression have been linked to initiation and progression of gliomas [17].

Distributional dimensions of increasing potentiality arise from the attributes of acquired stem cell profiles that malignantly transform. The whole spectrum of such distributional phenomenon indicates the institution of abnormal gene transcription consequent to both genetic and epigenetic influence.

Stem cell biology is central to a series of transformational events that primarily implicate the subventricular zone and which is further attributable to neurogenesis and particularly to systems of initial organogenesis. It is in view of parameters of incremental nature that the malignant transformation process is derived from stem-cell attributes of cancerization. miR-145 represses pluripotency of embryonic stem cells and is a tumor suppressor in different cancers [18].

The differential distinction of stemness includes an essential modulatory role in profile definition of glioma cells that is centered on the angiogenesis accompanying systems of field cancerization. Further to the shaping of stem cell derivation is the assumption of parameters of 
progression that add to the malignant potential for spread.

\subsection{Biologic Derivation}

Significant to the biologic derivation of cancer stem cells there evolves a mixture of attributes that are directly related to the onset of the malignant transformation process. Platelet-derived growth factor and c-Kit (stem cell factor receptor) signalling play important roles in initiation and maintenance of gliomas [19]. Especially prominent is the repeated re-characterization of systems of dedifferentiation that alternate with attempts at partial differentiation of progeny glioma cells. In such manner, the overall profiles overlap both stem cell biology and also organogenesis in terms of significant increase in potentiality for cell proliferation in particular.

The failure of current therapies has been attributed to the functional heterogeneity of glioma cells [20].

A relatively quiescent subset of glioma cells resembling cancer stem cells is responsible for long-term growth through the production of transient populations of highly proliferative cells [21].

The dimensions of stem cell biology are assumed reappraisal of the micro-environmental conditioning that modulates pathway activity and systems of consequential dedifferentiation.

Multi-lineage potential includes a specific role for cancer stem cells, in particular, that augment the range of distributional patterns for involvement of surrounding neural tissue by infiltrating tumor cells. Tumor-initiating cells might constitute most of the cells within the tumor bulk, and implicate a "hierarchical" model of tumorigenesis [22]. The whole complex derivation of mutagenesis is a consequence to the promotion for further dedifferentiation. The strict cooperative roles of stem cells are a significant component attribute in delineating potentiality for spread and tissue infiltration.

\subsection{Stemness}

The niche micro-environment is a site of microvascular angiogenesis in terms particularly of glioma initiation. Multi-directional modulatory influences account for the heterogeneity of many glioblastomas that promotes integral development of the neoplastic lesion.

The glioma cells, normal brain cells, immune cells, and stem cells interact through a complex cytokine network [23].

The concept of stemness is significant as proportional increment of the infiltration and multi-focal gliomagenesis that further promote in turn angiogenesis in adjacent fields of cancerization. Glioma stem cells induce the synthesis of vascular endothelial growth factor [24]. The profiles of neoplastic growth come to induce non-apoptosis and increased oncogene activity such as that of CDKN
2A. Mutation of suppressor genes such as TP53 allows a permissive assumption of excessive proliferative rate as integral to an overall dedifferentiation of cells and pathways.

Niche biology is a resource for cancerization and is promotional in terms of distribution dynamics in tumor modulation and spread. Overall dimensions of subsequent development and transformation assume the modulation of the micro-environment in the consequential progression of carcinogenesis or gliomagenesis. Perivascular and intraparenchymal tumor cell migration precede tumor mass formation in the adult brain [25].

Systems of repair are themselves also a source for the constitution of a neoplasm that is highly infiltrative. The significance of parameters of institution implicates dynamic modulators for the integral carcinogenesis as reflected in the development of various characterizations of putative stem cell activity.

The intraventricular route can achieve a reservoir of self-renewal cells, potentially active against glioma cell spread [26].

The development of injury is particularly prominent in the propagation of gliomagenesis in various foci of the cancerization field. It is the appearance of significant overlap of profiles of activity in such tumor fields that integrally come to constitute derivational dynamics of progressive growth and spread of the glioma cells.

Complex overlapping profiles of mutagenesis, radiation and oxidative stress culminate in a field effect that centers particularly on the stem cell niche. Dedifferentiation and stem cell attributes are systems of integral constitution in the delineation of events that are modulated by the micro-environment and in turn also modulate such micro-environment.

Promotional enhancement of gliomagenesis appears to contribute to the essential attributes of a lesion that is monoclonal, oligoclonal and also heterogeneous in many of its aspects in biological evolution. Identical tumorigenic stimuli produce markedly different glioma phenoltypes, depending on the differentiation status of the transformed cell [27]. The stem cells assume cancer attributes in their own right and beyond the defining characterization of the glioma cells themselves. It is further to the realization of a lesion of progressive nature that gliomas come to assume the multi-potentiality of the cancer stem cell that further conform to the disequilibrium of oncogenes with suppressor gene activity.

\section{DISCUSSION}

The developmental factors of gliomagenesis are dimensional systems of infiltrative activity beyond the parent tumor. Stem cells and cancer stem cells are end-results in their own right and contribute to a correlative interaction 
between cells and micro-environment. One major goal is targeting tumor antigens highly expressed on glioblastoma cancer stem cells [28].

The distribution of mutagenesis is a component system of correlative phenomena that enhance proliferative rate of gliomas within an essential modulation of the microenvironment. There is dynamic interplay among extracellular signal-regulated kinase, $\mathrm{N}$-cadherin and integrin alpha6 in glioma stem cell invasion [29].

Transitions of circulating stem cells through endothelial cells of the micro-vasculature account for the progression of fields of operative cancerization. It is significant to view complex re-modulation systems that expand the infiltrative field of a glioma within the contextual reference of further enhancement of the malignant transformation zone. Oxidative stress linked to such pathways as metallotheonins would allow for a transformation that is both parent to the excessive proliferative rate of glioma cells and also to spread within the field of cancerization.

Duality of roles in cancerization involves the establishment of gliomagenesis that participates in the overall development of foci of collaborative infiltration.

Tumor-associated microglia/macrophages may also play important roles in promoting glioma stem cell invasion, particularly via transforming growth factor-Beta1 release [30]. The malignant transformation process is due to cancer stem cell establishment in various dynamic activities that implicate response to genetic and epigenetic change and the infiltration of tumor cells relative to foci of angiogenesis. Hypoxia is the main micro-environmental conditioning mechanism that parallels the gliomagenesis with angiogenesis. The field cancerization phenomenon is secondarily implicated in ways that are potentiated by the onset of systems of genetic and epigenetic factors of injury [31].

It is with reference to various simultaneous events that the sequential conditioning of stem cell niches proves a progressive development of carcinogenesis. There is a need for integration of models of cancer as an organ system in the development of cancer therapies [32].

Incremental evolutionary traits hence are dedifferentiating in terms of the defining roles of cancer stem cells. In view of increased proliferative rate, and also the characterization of developmental traits, delineation of considerable heterogeneity occurs within any given glioma that is participant to multiple consensus development of concomitant and sequential events of interaction between cells and cells and between cells and the micro-environment. In this regard, the system realization of injury is strictly and potentially definable in terms beyond the simple end-result of further incremental infiltration of the surrounding brain tissues.

It is to be realized that definition of the role of malig- nant change as end-result of cancer stem cell biological activity would imply a stemness that is conceptually activated within the conditioning parameters of the niche micro-environment.

The micro-circulatory system of stem cell niches delineates the sharp distinction between neural stem cells and the cancer stem cells. Initiation and further progresssion allow for permissive conditions to further characterize the realization of a whole plethora of injuries as carcinogenesis. Glioma stem cells aberrantly differentiate into diverse cell types and may contribute to the establishment of tumor heterogeneity [33].

The differentiation process of transformed malignant change is attributed to the defining properties of a series of dynamic interactions as shown by the inception of fields of carcinogenesis. Patch characterization includes the pushing of surrounding neural tissues, while the foci of tumor transformation expand. Collision parameters of multiple foci of gliomagenesis allow for incremental development of injury as defined oncogenically.

Angiogenesis is a standard structural marker of injury that allows for permissive conditions of realized potentiality and the possible occurrence of recurrence and for the appearance of therapy resistance.

The whole spectrum of participating influences are conditioning pathways as foci of malignant transformation that concurrently promote niches that harbor cancer stem cells.

Hence, it is the niche micro-environment that is established influence in gliomagenesis. In a last analysis, it is beyond the simple acquisition of genetic damage that tumor or glioma cells prove progressive as infiltration of fields of established genetic injury. Markers or glioblastoma stem cells such as CD133, CD15, integrin alpha6, L1CAM may aid identification but cannot be conclusively linked to a stem cell phenotype [34].

\section{CONCLUSIONS}

Delineation of genetic injury is a karyotypic marker of malignant transformation in a manner that is permissive in the initial stages of gliomagenesis. It is further to such injury that the fields of cancerization allow for conditioned niches to promote the replication of cancer stem cells in close relative proximity to niche micro-vasculature.

The significance of further interplay between transforming cells and micro-environmental conditioning allows for the emergence of stem cells that contribute to the establishment of a series of overlapping profiles that incrementally participate also as qualitative characterizing traits of the transition between neural stem cells and cancer stem cells. Realization of oncologic onset of the lesion is therefore a concurrently evolving series of pathways that molecularly prove accumulative. 
It is further to the dimensional occurrence of excessive proliferative activity and of micro-environmental infiltration that the consequential attributes of pathway involvement are both transforming in qualitative terms and also quantitative in lesion dynamics.

\section{REFERENCES}

[1] Bazzoli, E., Pulvirenti, T., Oberstedt, M.C., Perna, F., Wee, B., Schultz, N., et al. (2012) MEF promotes stemness in the pathogenesis of glioma cell. Stem Cell, 11, 836-844. doi:10.1016/j.stem.2012.09.012

[2] Tan, J., Meng, Y., Huang, S. and Wang, P. (2012) Therapeutic gene products delivery by neuron stem cells. Current Pharmaceutical Biotechnology, 13, 2427-2431. doi:10.2174/138920112803341806

[3] Kong, B.H., Park, N.R., Shim, J.K., Kim, B.K., Shin, H.J., Lee, J.H., et al. (2012) Isolation of glioma cancer stem cells in relation to histological grades in glioma specimens. Child's Nervous System, 10 November 2012. doi:10.1007/s00381-012-1964-9

[4] Ho, I.A., Toh, H.C., Ng, W.H., Teo, Y.L., Guo, C.M., Hul, K.M., et al. (2012) Human bone-marrow-derived mesenchymal stem cells suppress human glioma growth through inhibition of angiogenesis. Stem Cells, 31, 146-155.

[5] Ueda, Y., Wei, F.Y., Hide, T., Michiue, H., Takayama, K., Kaitsuka, T., Nakamura, H., et al. (2012) Induction of autophagic cell death of glioma-initiating cells by cellpenetrating d-isomer peptides consisting of Pas and the p53C-terminus. Biomaterials, 33, 9061-9069. doi:10.1016/j.biomaterials.2012.09.003

[6] Zou, Y., Niu, W., Qin, S., Downes, M., Burns, D.K. and Zhang, C.L. (2012) The nuclear receptor TLX is required for gliomagenesis within the adult neurogenic niche. Molecular and Cellular Biology, 32, 4811-4820. doi:10.1128/MCB.01122-12

[7] Lee da, Y., Gianino, S.M. and Gutmann, D.H. (2012) Innate neural stem cell heterogeneity determines the patterning of glioma formation in children. Cancer Cell, 22, 131-138. doi:10.1016/j.ccr.2012.05.036

[8] Wu, N., Xiao, L., Zhao, X., Zhao, J., Wang, J., Wang, F., et al. (2012) MiR-125b regulates the proliferation of glioblastoma stem cells by targeting E2F2. FEBs Letters, 586, 3831-3839.

[9] de Alucida Sassi, F., Lunardi Brunetto, A., Schwartsmann, G., Roesler, R. and Abujamra, A.L. (2012) Glioma revisited: From neurogenesis and cancer stem cells to the epigenetic regulation of the niche. Journal of Oncology, 2012, 537861.

[10] Kim, S.M., Woo, J.S., Jeong, C.H., Ryu, C.H., Lim, J.Y. and Jeun, S.S. (2012) Effective combination therapy for malignant glioma with TRAIL-secreting mesenchymal stem cells and Lipoxygenase Inhibitor MK886. Cancer Research, 72, 4807-4817. doi:10.1158/0008-5472.CAN-12-0123

[11] Dokic, I., Hartmann, C., Herold-Mende, C. and RegnierVigouroux, A. (2012) Glutathione peroxidase 1 activity dictates the sensitivity of glioblastoma cells to oxidative stress. Glia, 60, 1785-800. doi:10.1002/glia.22397

[12] Kim, H.S., Woolard, K., Lai, C., Bauer, P.O., Maric, D., Song, H., et al. (2012) Gliomagenesis arising from Ptenand Ink 4a/Arf-deficient neural progenitor cells is mediated by the p53-Fbxw 7/Cdc4 pathway, which controls c-Myc. Cancer Research, 72, 6065-6075. doi:10.1158/0008-5472.CAN-12-2594

[13] Gopisetty, G., Xu, J., Sampath, D., Colman, H. and Puduvalli, V.K. (2012) Epigenetic regulation of CD133/PROM 1 expression in glioma stem cells by Sp1/myc and promoter methylation. Oncogene, 3 September 2012.

[14] Charles, N.A., Holland, E.C., Gilbertson, R., Glass, R. and Kettenmann, H. (2012) The brain tumor microenvironment. Glia, 60, 502-514.

[15] Lima, F.R., Kahn, S.A., Soletti, R.C., Biasoli, D., Alves, T., daFonseca, A.C., et al. (2012) Glioblastoma: Therapeutic challenges, what lies ahead. Biochimica et Biophysica Acta, 1826, 338-349.

[16] Cruz, M.H., Siden, A., Calaf, G.M., Delwar, Z.M., Yakisich, J.S. (2012) The stemness phenotype model. ISRN Oncology, 2012, 392647.

[17] Chan, X.H., Nama, S., Gopal, F., Rizk, P., Ramasamy, S., Sundarain, G., et al. (2012) Targeting glioma stem cells by functional inhibition of a Prosurvival OncomiR-138 in malignant gliomas. Cell Reports, 2, 591-602. doi:10.1016/j.celrep.2012.07.012

[18] Speranza, M.C., Frattini, V., Pisati, F., Kapetis, D., Porrati, P., Eoli, M., et al. (2012) NEDD9, a novel target of miR-145, increases the invasiveness of glioblastoma. Oncotarget, 3, 123-134.

[19] Dong, Y., Han, Q., Zou, Y., Deng, Z., Lu, X., Wang, X., et al. (2012) Long-term expsure to imatinib reduced cancer stem cell ability through induction of cell differentiation via activation of MAPK signalling in glioblastoma cells. Molecular and Cellular Biochemistry, 370, 89-102. doi:10.1007/s11010-012-1401-0

[20] Jiang, Y. and Uhrborn, L. (2012) On the origin of glioma. Upsala Journal of Medical Sciences, 117, 113-121. doi:10.3109/03009734.2012.658976

[21] Chen, J., Li, Y., Yu, T.S., McKay, R.M., Burns, D.K., Kernie, S.G., et al. (2012) A restricted cell population propagates glioblastoma growth after chemotherapy. $\mathrm{Na}$ ture, 488, 522-526. doi:10.1038/nature11287

[22] Mazzoleni, S. and Galli, R. (2012) Gliomagenesis: A game played by few players or a team effort. Frontiers in Bioscience, 4, 205-213.

[23] Zhu, V.F., Yang, J., Lebrun, D.G. and Li, M. (2012) Understanding the role of cytokines in glioblastoma multiforme pathogenesis. Cancer Letters, 316, 139-150. doi:10.1016/j.canlet.2011.11.001

[24] Bulnes, S., Bengoetxea, H., Ortuza, N., Argandona, E.G., Garcia-Blanco, A., Rico-Barrio, I., et al. (2012) Angiogenic signalling pathways atered in gliomas; selection mechanisms for more aggressive neoplastic subpopulations with invasive phenotype. Journal of Signal Transduction, 2012, 597915.

[25] Sampetrean, O., Saga, I., Nakanishi, M., Sugihara, E., 
Fukaya, R., Onishi, N., et al. (2011) Invasion precedes tumor mass formation in a malignant brain tumor model of genetically modified neural stem cells. Neoplasia, 13, 784-791.

[26] Panciani, P.F., Fontanella, M., Tamagno, I., Battaglia, L., Garbossa, D., Inghirami, G., et al. (2012) Stem cells based therapy in high grade glioma: Why the intraventricular route should be preferred? Journal of Neurosurgical Sciences, 56, 221-229.

[27] Ghazi, S.O., Stark, M., Zhao, Z., Mobley, B.C., Munden, A., Hover, L., et al. (2012) Cell of origin determines tumor phenotype in an oncogenic Ras/p53 knockout transgenic model of high grade glioma. Journal of Neuropathology \& Experimental Neurology, 71, 729040. doi:10.1097/NEN.0b013e3182625c02

[28] Phuphanich, S., Wheeler, C.J., Rudnick, J.D., Mazer, M., Wang, H., Nuno, M.A., et al. (2012) Phase 1 Trial of a multiepitope-pulsed dendritic cell vaccine for patients with newly diagnosed glioblastoma. Cancer Immunology, Immunotherapy, Epub Ahead of Print. doi:10.1007/s00262-012-1319-0

[29] Velpula, K.K., Rehman, A.A., Chelluboina, B., Dasari, V.R., Gondi, C.S., Rao, J.S., et al. (2012) Glioma stem cell invasion through regulation of the interconnected ERK, integrin alpha6 and N-cadherin signalling pathway.
Cell Signal, 24, 2076-2084. doi:10.1016/j.cellsig.2012.07.002

[30] Ye, X.Z., Xu, S.L., Xin, Y.H., Yu, S.C., Ping, Y.F., Chen, L., et al. (2012) Tumor associated microglia/macrophages enhance the invasion of glioma stem-like cells via TGFBeta1 Signaling pathway. Journal of Immunology, 189, 444-453. doi:10.4049/jimmunol.1103248

[31] Florio, T. and Barbieri, F. (2012) The status of the art of human malignant glioma management: The promising role of targeting tumor-initiating cells. Drug Discovery Today, 17, 1103-1110 doi:10.1016/j.drudis.2012.06.001

[32] Kim, Y., Kim, E., Wu, Q., Guryanova, O., Hitomi, M., Lathia, J.D., et al. (2012) Platelet-derived growth factor receptors differentially inform intertumoral and intratumoral heterogeneity. Genes Development, 26, 1247-1262. doi:10.1101/gad.193565.112

[33] Katsushima, K., Shinjo, K., Natsume, A., Ohka, F., Fujii, M., Osada, H., et al. (2012) Contribution of microRNA1275 to Claudin 11 protein suppression via a polycombmediated silencing mechanism in human glioma stemlike cells. The Journal of Biological Chemistry, 287, 27396-27406. doi:10.1074/jbc.M112.359109

[34] Brescia, P., Richichi, C. and Pelicci, G. (2012) Current strategies for identification of glioma stem cells: Adequate or unsatisfactory? Journal of Oncology, 2012, 376894. 